\title{
Classroom activities to study Magnetic field patterns due to current configurations
}

\author{
"Raveesha K.H ${ }^{1}$, Vedavathi $\mathbf{P}^{2}$, Vijayakumar H Doddamani ${ }^{3}$ \\ ${ }^{1}$ Department of Physics, CMR Institute of Technology, Bangalore -560037, India \\ ${ }^{2,3}$ Department of Physics, Bangalore university,Bengaluru-560056, India \\ hod.physics@cmrit.ac.in
}

E-mail: hod.physics@cmrit.ac.in

\begin{abstract}
Magnets are widely used in electric devices such as motors, generators, speakers, microphones, scanning devices etc. Magnetic field produced by a current configuration like solenoid, circular coil and straight conductor find applications in engineering. In this article, techniques of visualizing the magnetic field patterns due to the above current configurations is demonstrated. These experiments are beneficial to identify suitable current configurations to produce a specific field pattern for applications in scanning and other applications. These experiments can be easily conducted in classroom. Students acquire practical knowledge on relationship between current and magnetic fields. This enables them to inculcate application skills to design current distributions to obtain a specific magnetic field pattern.
\end{abstract}

Keywords: Magnetic field, Solenoid, Circular coil

\section{Introduction:}

Magnetic field pattern of a Bar magnet is the most common picture seen in text books of Physics in schools and Junior colleges. Similar picture for current carrying coils is also shown. However, experiments to relate magnetic field pattern with current configuration are not a regular feature in a classroom [1][2[5]]. Authors in their two decade experience in teaching school and undergraduate students have noticed that students in general lack application skills in visualizing magnetic field pattern due to specific current distribution.

In this paper, simpler techniques of magnetic field visualization due to straight conductor, Circular coil and Solenoid are described. Authors have observed that these activities make students to critically think on the nature of magnetic fields.

\section{Techniques}

We present here three activities performed using items available in a normal laboratory. The coils used are manually wound. Regulated power supply (0-30V, 0-3A) is used as Direct current (D.C) source.

\subsection{Magnetic Field Due To Straight Conductor}

Current in a straight conductor causes magnetic flux lines in a plane perpendicular to current. The experimental setup comprises rectangular plastic frame over which 22 gauge copper wire is wound with around 100 turns as shown in figure $1 \& 2$. A cardboard is fixed perpendicular to the current direction and thin iron powder is sprinkled on it. A current of $0.25 \mathrm{~A}$ and voltage $25 \mathrm{~V}$ is applied to this circuit. The card board is gently tapped and the fine iron powder particles align along the circular magnetic flux lines. The magnetic field lines are in a plane perpendicular to direction of current [4]. Magnetic field intensity may be determined by comparing this pattern with calibrated field patterns.

\subsection{Magnetic Field Due To Circular Coil}

Current in a circular coil causes magnetic flux lines in a plane perpendicular to current and entering into the plane of the coil. The experimental setup consists of a circular coil with 22 gauge copper wire having around 500 turns as shown in figure $3 \& 4$. A cardboard is fixed perpendicular to the plane of the coil. 


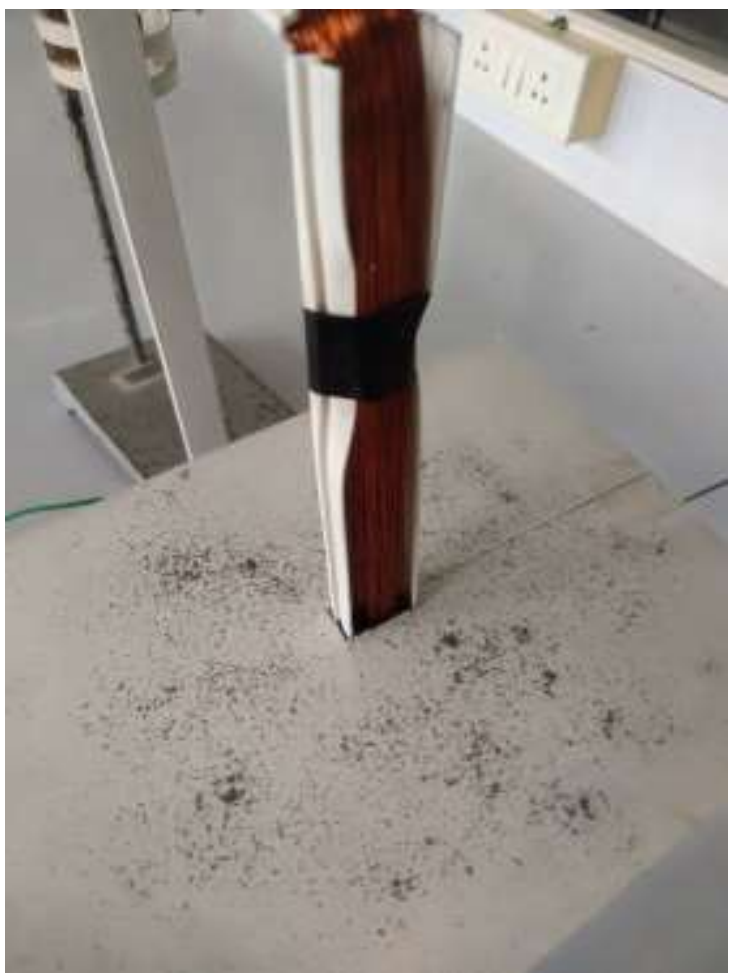

Figure 1 A straight conductor without current flow. Note random distribution of Iron powder

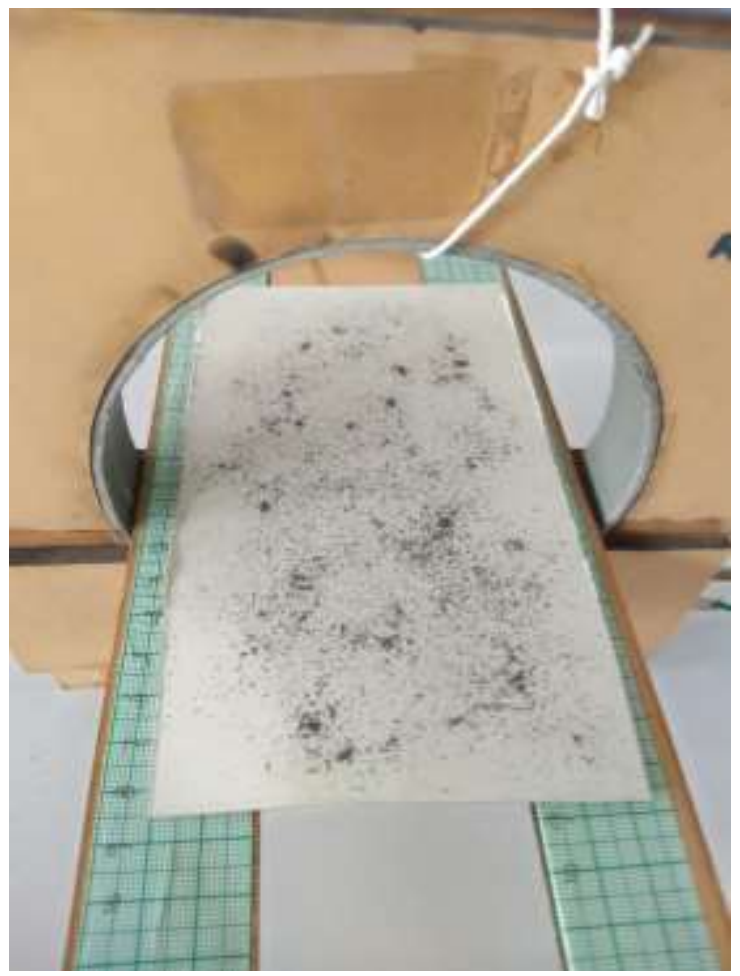

Figure2 A circular coil without current flow

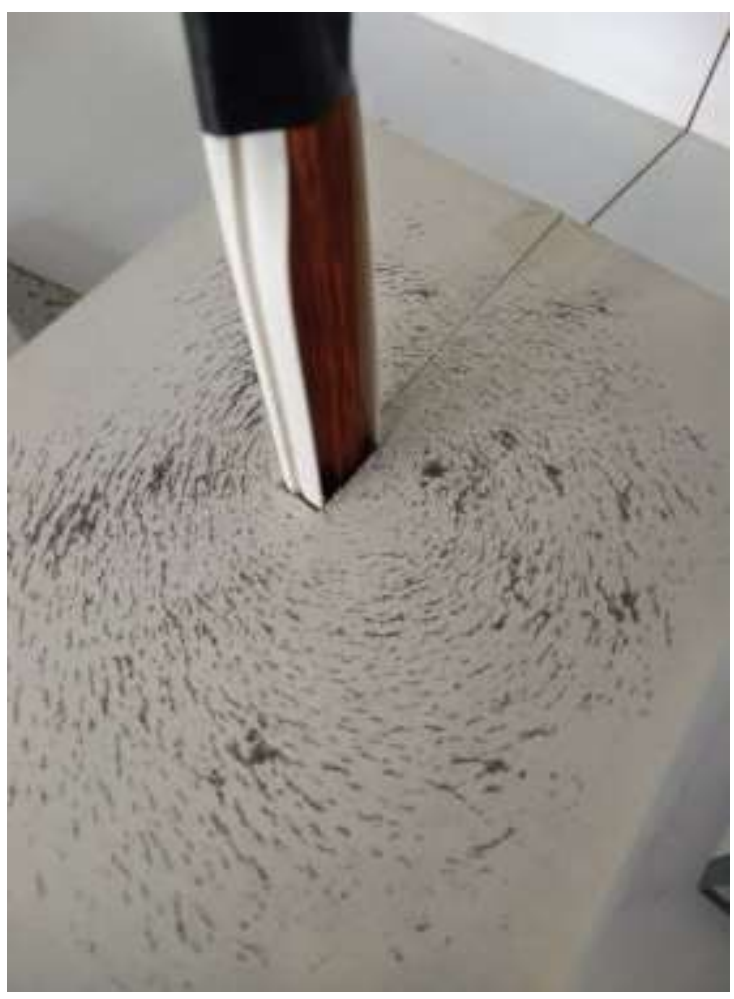

Figure 2 Magnetic field pattern for a straight conductor with current flow. Observe alignment of fine Iron powder along circular field lines

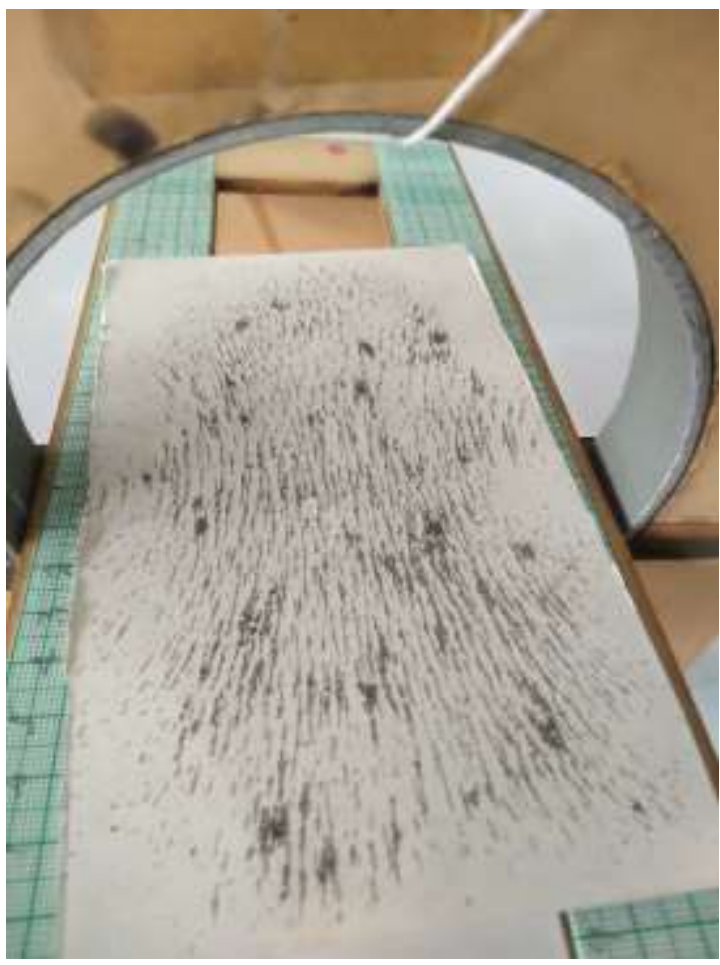

Figure 3 Magnetic field pattern for circular coil with current. Iron powder particles align along the Field lines moving into/away from the plane of the coil 


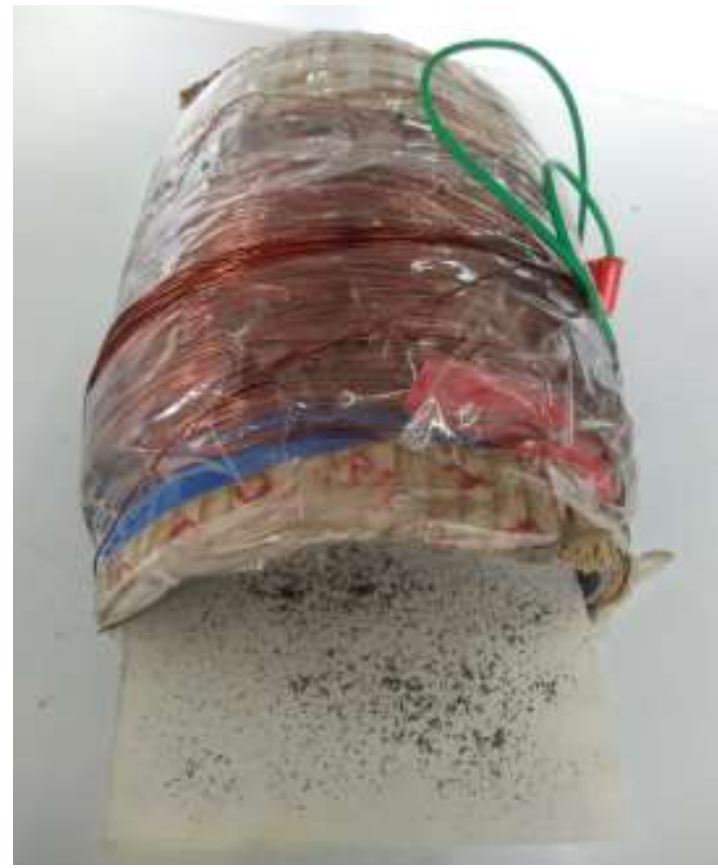

Figure 5 Solenoid without current flow

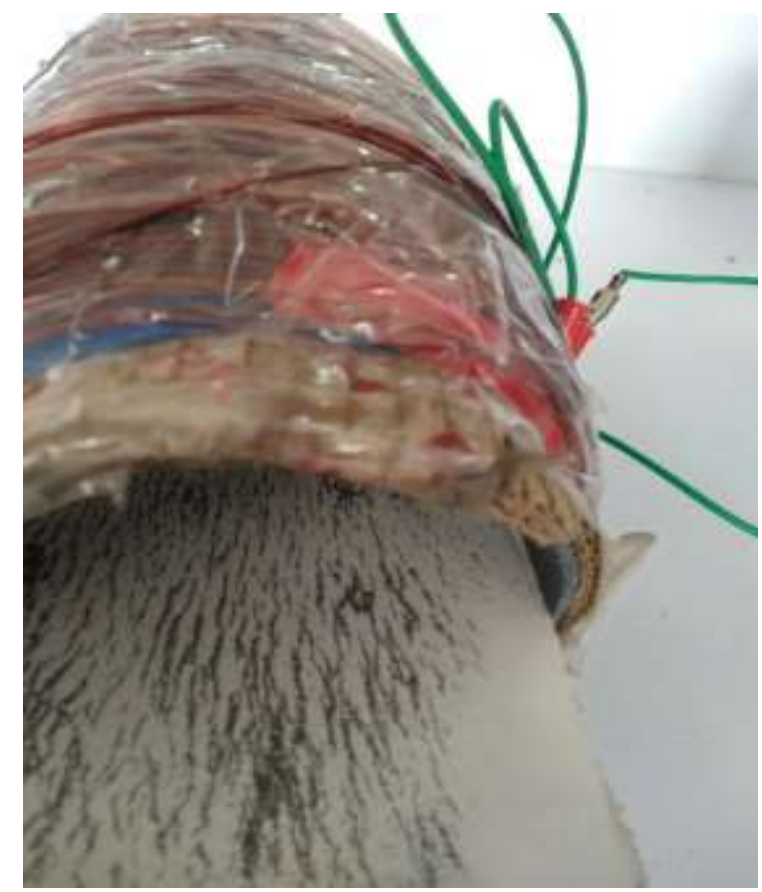

Figure 6 Magnetic field pattern for a solenoid with current. Iron powder aligned along the axial field lines

Thin iron powder is sprinkled on it. A current of $0.25 \mathrm{~A}$ and voltage $25 \mathrm{~V}$ is applied to this circuit. The card board is gently tapped and the Iron powder particles align along magnetic flux lines. These field lines either move either onto or away from the plane of the coil [3]. Magnetic field Intensity may be determined by comparing this pattern with calibrated field patterns.

\subsection{Magnetic Field Due To Solenoid}

Current in a circular solenoid causes magnetic flux lines along the axis of the coil [3]. The experimental setup consists of a circular coil with 22gauge copper wire having around 2000 turns as shown in figure 5 \& 6.A cardboard is fixed along the axis of the coil. Fine Iron powder is sprinkled on it. A current of $0.25 \mathrm{~A}$ and voltage $25 \mathrm{~V}$ is applied to this circuit. The card

board is gently tapped and the Iron powder particles align along the magnetic flux lines along the axis. This experiment shows that field pattern for a solenoid resembles that of a bar magnet [4]. Magnetic field intensity may be determined by comparing this pattern with calibrated field patterns.

\section{Conclusions}

The field patterns observed in the above techniques are of high clarity and can be utilized to determine the magnetic field intensity using standard calibration tools. These activities can be introduced at the Precollege level itself. This activity introduces students to magnetic field generation, its effects. Students may be encouraged to extend these ideas to visualize magnetic field patterns in major applications like particle accelerators, scanning techniques such as Magnetic Resonance Imaging (MRI). This motivates students to take part in research activities and develop critical thinking, analytical skills. These methods being simple and also can be performed with low cost tools available in a laboratory have advantages over the other techniques.

\section{Acknowledgements}

We thank authorities of CMR Institute of Technology, Bengaluru and Bangalore university, Bangalore for providing assistance to carry out this research work. We express our gratitude to our colleague Dr Rajesh Gopal, laboratory staff Mr. Lingaswamy for their help in setting up of this experiment. Our thanks are due to referee for the suggestions. 


\section{References}

1. Aungtinee Kittiravechote, European J of Physics Education Volume 11 Issue 1 1309-7202

2. Cai Wei et al 2019 J. Phys.: Conf. Ser. 1237032073

3. David K Cheng 1983 Electromagnetics (California : Addison - Wesley )

4. D Chattopadhyay and P C Rakshit 2005 An advanced course in Practical Physics ( Kolkata : Central)

5. Miloš Babović and Vukota Babović 2017 Phys. Educ. 52015021 